\title{
Quantitative analysis of protein crotonylation identifies its association with immunoglobulin A nephropathy
}

\author{
HUA LIN $^{1}$, DONGE TANG ${ }^{2}$, YONG XU ${ }^{2}$, RUOHAN ZHANG $^{1}$, MINGLIN OU $^{1}$, \\ FENGPING ZHENG $^{2}$, JIEJING CHEN ${ }^{1}$, YUE ZHANG ${ }^{1}$, GUIMIAN ZOU $^{1}$, WEN XUE ${ }^{1}$, \\ YAOSHUANG ZOU ${ }^{1}$, WEIER DAI ${ }^{3}$, WEIGUO SUI ${ }^{1}$ and YONG DAI ${ }^{1,2}$
}

\begin{abstract}
${ }^{1}$ Nephrology Department of 924th Hospital, Guangxi Key Laboratory of Metabolic Diseases Research, Guilin Key Laboratory of Kidney Diseases Research, Guilin, Guangxi 541002; ${ }^{2}$ Clinical Medical Research Center of The Second Clinical Medical College of Jinan University, Shenzhen People's Hospital, Shenzhen, Guangdong 518020, P.R. China; ${ }^{3}$ College of Natural Science, University of Texas at Austin, Austin, TX 78712, USA
\end{abstract}

Received June 9, 2019; Accepted November 19, 2019

DOI: $10.3892 / \mathrm{mmr} .2020 .10931$

\begin{abstract}
Posttranslational modifications (PTMs) to histones such as lysine crotonylation are classified as epigenetic changes. Lysine crotonylation participates in various cellular processes and occurs in active promoters, directly accelerating transcription. The present study performed a proteomics analysis of crotonylation between healthy controls and patients with immunoglobulin A (IgA) nephropathy using tandem mass spectrometry and high-resolution liquid chromatography. The present results identified 353 crotonylated proteins and 770 modification sites, including 155 upregulated and 198 downregulated crotonylated proteins. In total, seven conserved motifs were identified in the present study. The present bioinformatics analysis results suggested a number of the crotonylated proteins exhibited various subcellular localization patterns, such as in the cytoplasm. Protein domains, including thioredoxin, moesin tail and myosin like IQ motif domains were markedly enriched in crotonylated proteins. Kyoto Encyclopedia of Genes and Genomes and functional enrichment analyses suggested significant enrichment of crotonylated proteins in complement and coagulation cascades, and antigen processing and presentation pathways displaying important relationships with IgA nephropathy. The present results suggested that crotonylation occurred in numerous proteins and may play key regulatory roles in $\operatorname{Ig} \mathrm{A}$ nephropathy.
\end{abstract}

Correspondence to: Professor Yong Dai or Mr. Weiguo Sui, Nephrology Department of 924th Hospital, Guangxi Key Laboratory of Metabolic Diseases Research, Guilin Key Laboratory of Kidney Diseases Research, 1 Xinqiaoyuan Road, Xiangshan, Guilin, Guangxi 541002, P.R. China

E-mail: daiyong22@aliyun.com

E-mail: suiwg@163.com

Key words: posttranslational modifications, lysine crotonylation, tandem mass spectrometry, proteome, immunoglobulin A nephropathy

\section{Introduction}

Posttranslational modifications (PTMs) have important roles in numerous biological processes, including organismal development and cell differentiation, thus constituting a primary pathway in epigenetic regulation (1). PTMs of proteins have been discovered using various technologies, including mass spectrometry (MS) (2). PTMs present at low levels are enriched at the peptide level in MS detection $(3,4)$. Using MS-based proteomics, the present study may have identified novel histone PTMs, such as crotonylation and different non-acetyl lysine acylation events. Lysine crotonylation regulates the transcription of active genes in the murine male germ cell and human cell genomes (5). Furthermore, lysine crotonylation may play an important role in modulating gene expression in male haploid cells (6). Lysine crotonylation is principally related to active transcription (7). The histone acetyltransferase p300/CREB-binding protein catalyzes histone crotonylation and directly participates in transcriptional activation in vivo (8). Moreover, the abundance of crotonyl-coenzyme A may affect levels of crotonylation (9). Therefore, lysine crotonylation sites in proteins must be identified to provide improved understanding of the physiological functions of crotonylation.

PTMs have been identified in subjects with chronic renal disease (10). Crotonylation has been identified in renal tissues and is a key contributor to the epigenetic regulation of gene expression (5). A useful in vivo effect over preclinical kidney injury has been showed for drugs that interfere with histone modification readers such as inhibition of extra-terminal proteins and bromodomain, or increase histone crotonylation (11). Histone crotonylation protects against nephrotoxic acute kidney injury; however, research is inconsistent on DNA methylation inhibitors for preclinical acute kidney injury (12). Immunoglobulin A nephropathy (IgAN), a common glomerular disease that results in chronic or end-stage kidney disease (13), is caused by an accumulation of IgA in the glomerular mesangium (14). Differences in IgAN have been observed between ethnicities, with a higher incidence disease reported in Asian populations (15). A high concentration of 
IgA in the blood increases the risk of $\operatorname{IgAN}(16)$. Patients are diagnosed with IgAN or other glomerulonephritides by a kidney biopsy, which is an invasive method (17). Therefore, non-invasive diagnostic markers of IgAN would aid current laboratory and clinical methods. In total, $30 \%$ of patients with $\mathrm{Ig} \mathrm{AN}$ progress to end-stage renal disease, with the remainder having low-grade proteinuria or hematuria (18). The pathogenic mechanisms of $\operatorname{Ig} \mathrm{AN}$ are complex. IgA immune complexes, including the IgA1 subtype, accumulate in the glomerulus, and cause inflammation and renal injury (19). A previous study revealed a new function for microRNAs (miRs) in nephropathy with IgA1 (20). Abnormal miR-148b expression results in high levels of galactose-deficient $\operatorname{IgA} 1$ in peripheral blood mononuclear cells (PBMCs) in patients with IgAN (21). Additional factors such as CD89-IgA complexes and low-molecular-weight proteins in the blood are related to hematuria, proteinuria and organic lesions $(22,23)$. However, no previous studies have translated into clinical assessment, thus the specificity of the findings for IgAN must be evaluated. Currently, genetic and serological methods for differentiating IgAN from other renal diseases are unavailable. Therefore, the pathogenesis of glomerular disease must be investigated.

The present study performed liquid-chromatography fractionation and MS to investigate differences in crotonylated peptides and proteins between healthy negative controls (NC) and patients with IgAN. Subsequently, bioinformatics analysis was conducted to investigate the functions of markedly enriched proteins. Crotonylation was identified in a proteomics analysis of samples from patients with IgAN. The aim of the present study was to understand the function of lysine crotonylation in patients with IgAN. PTMs may represent a potentially novel biomarker and factor contributing to the pathogenesis of $\operatorname{Ig} \mathrm{AN}$.

\section{Materials and methods}

Controls and patients. Peripheral blood was collected from 6 patients at 924nd Hospital between March 2017 and April 2018 (age, 25-41, 33 \pm 8 ; male to female ratio of 4:2) with IgAN and 25 control subjects (age 30-50, 38 \pm 4.5 ; male to female ratio of 16:9). The diagnosis of $\operatorname{IgAN}$ in all patients was confirmed via histology. IgAN was diagnosed based on the presence of dominant $\operatorname{IgA}$ deposits in proliferating mesangial cells and glomerular mesangial cells (24). Laboratory tests were also performed to determine the creatinine expression level $(236.5 \pm 250.27)$ by the sarcosine oxidase end point method (25) (Zhejiang Kuake Biotechnology Co., Ltd.) and the proteinuria level $(1.79 \pm 1.55)$ by the pyrogallol red molybdenum one point terminal method (26) (Zhejiang Kuake Biotechnology Co., Ltd.). Informed consent was obtained from all participants. The study was approved by The 924 nd Hospital Ethics Committee and conducted in compliance with the Declaration of Helsinki.

PBMC isolation and protein extraction. A 10-ml blood sample was collected from each participant into heparinized vacutainers. PBMCs were obtained via density gradient centrifugation at $1,000 \times \mathrm{g}$ for $10 \mathrm{~min}$ at room temperature with Hypaque-Ficoll (GE Healthcare Life Sciences), lysed using TRIzol ${ }^{\circledR}$ reagent (Invitrogen; Thermo Fisher Scientific,
Inc.) and stored at $-80^{\circ} \mathrm{C}$. Samples were sonicated 3 min at $4^{\circ} \mathrm{C}$ three times on ice using a high intensity ultrasonic processor (Ningbo Scientz Biotechnology Co., Ltd.) in lysis buffer containing $8 \mathrm{M}$ urea (Sigma-Aldrich; Merck KGaA) and 1\% protease inhibitor cocktail (Merck KGaA). The remaining debris was centrifugated at $12,000 \mathrm{x}$ for $10 \mathrm{~min}$ at $4^{\circ} \mathrm{C}$. The supernatant was obtained and the protein concentration in all six IgAN samples was determined using a BCA kit.

Trypsin digestion. The protein solution was reduced with $5 \mathrm{mM}$ dithiothreitol for $30 \mathrm{~min}$ at $56^{\circ} \mathrm{C}$ and alkylated with $11 \mathrm{mM}$ iodoacetamide for $15 \mathrm{~min}$ at room temperature in the dark. The protein sample was then diluted with $100 \mathrm{mM}$ triethylammonium bicarbonate (TEAB) buffer (Sigma-Aldrich; Merck $\mathrm{KGaA}$ ) to obtain a urea concentration $<2 \mathrm{M}$. Trypsin was added at a 1:50 trypsin-to-protein mass ratio for the first digestion overnight at $37^{\circ} \mathrm{C}$ and a $1: 100$ trypsin-to-protein mass ratio for a second 4 -h digestion at $37^{\circ} \mathrm{C}$.

Tandem mass tag (TMT) labelling. Peptides were desalted using a Strata X C18 SPE column (Phenomenex) and then vacuum-dried. The peptides were reconstituted in $0.5 \mathrm{M}$ TEAB and processed with a TMT Mass Tagging kits and reagents (Thermo Fisher Scientific, Inc.). One unit of TMT reagent was thawed and reconstituted in acetonitrile (100\%). The peptide mixtures were incubated with the mixture for $2 \mathrm{~h}$ at room temperature, pooled, desalted and dried by vacuum centrifugation at $1,400 \mathrm{x}$ for $2 \mathrm{~h}$ at $30^{\circ} \mathrm{C}$.

High-performance liquid chromatography (HPLC) fractionation. No internal standards were used. The flow rate was $1 \mathrm{ml} / \mathrm{min}$. Column temperature box was $35^{\circ} \mathrm{C}$ during the classification. Mobile phase A: $98 \%$ water and $2 \%$ acetonitrile, Mobile phase B: $2 \%$ water and $98 \%$ acetonitrile. Tryptic peptides were fractionated using high $\mathrm{pH}$ reverse-phase HPLC (Agilent 1260; Agilent Technologies $\mathrm{GmbH}$ ) with a Thermo Betasil C18 column (Thermo Fisher Scientific, Inc.; particle size, $5 \mu \mathrm{m}$; ID, $10 \mathrm{~mm}$; length, $250 \mathrm{~mm})$. The peptides $(200 \mu \mathrm{g})$ were first separated into 60 fractions using a gradient of 8-32\% acetonitrile ( $\mathrm{pH} 9.0$ ) over $60 \mathrm{~min}$, combined into crotonylated fractions and dried via centrifugation under a vacuum at $1,400 \mathrm{x} \mathrm{g}$ for $5 \mathrm{~h}$ at $30^{\circ} \mathrm{C}$.

Affinity enrichment. Tryptic peptides dissolved in NETN buffer $(100 \mathrm{~mm} \mathrm{NaCl} ; 1 \mathrm{~mm}$ EDTA; $50 \mathrm{~mm}$ Tris- $\mathrm{HCl} ; 0.5 \%$ NP-40; pH 8.0) were incubated overnight with prewashed antibody beads (PTM Biolabs, Inc.) at $4^{\circ} \mathrm{C}$ with gentle shaking to enrich for crotonylated peptides. The beads were washed four times with NETN buffer and twice with $\mathrm{H}_{2} \mathrm{O}$. The bound peptides were eluted from the beads with $0.1 \%$ trifluoroacetic acid and the eluted fractions were combined and vacuum-dried. For liquid chromatography-MS (LC-MS/MS) analysis, the resulting peptides were desalted with C18 ZipTips (EMD Millipore).

LC-MS/MS analysis. Tryptic peptides were dissolved in $0.1 \%$ formic acid (solvent A) and directly loaded onto a homemade reverse-phase analytical column (length, $15 \mathrm{~cm}$; ID $75 \mu \mathrm{m}$ ). The gradient was comprised of an increase from 6 to $23 \%$ solvent B ( $0.1 \%$ formic acid in $98 \%$ acetonitrile) over $26 \mathrm{~min}$, 
23 to $35 \%$ in $8 \mathrm{~min}$, an increase to $80 \%$ in $3 \mathrm{~min}$ and then holding at $80 \%$ for the last $3 \mathrm{~min}$, all at a constant flow rate of $400 \mathrm{nl} / \mathrm{min}$ using an EASY-nLC 1000 ultra-performance liquid chromatography system (Thermo Fisher Scientific, Inc.). The peptides were subjected to the nanospray positive ionization source followed by MS/MS with a Q Exactive Plus instrument (Thermo Fisher Scientific, Inc.) coupled online to an ultra-performance liquid chromatograph. The electrospray voltage was $2.0 \mathrm{kV}$. The $\mathrm{m} / \mathrm{z}$ scan range was $350-1,800$ for a full scan and intact peptides were examined by an Orbitrap at a resolution of 70,000. Peptides were selected for MS/MS with a normalized collision energy setting of 28 , and the fragments were analyzed by the Orbitrap at a resolution of 17,500. A data-dependent procedure that alternated between one MS scan followed by $20 \mathrm{MS} / \mathrm{MS}$ scans with 15.0 -sec dynamic exclusion was performed. Automatic gain control was set to $5 \mathrm{E} 4$. The fixed first mass was set to $100 \mathrm{~m} / \mathrm{z}$.

Database search. MS/MS data were analyzed with the Maxquant search engine (v.1.5.2.8) (27). Tandem MS data were searched against the crotonylation database (https://www. uniprot.org/) concatenated with a reverse decoy database (27). Trypsin/P was specified as the cleavage enzyme and $\leq 4$ missed cleavages were allowed. The mass tolerance for precursor ions was set to $20 \mathrm{ppm}$ in the first search and $5 \mathrm{ppm}$ in the main search; the mass tolerance for fragment ions was set to $0.02 \mathrm{Da}$. Carbamidomethylation of cysteine was specified as the fixed modification, and crotonylation and oxidation of methionine were specified as variable modifications. The false discovery rate was adjusted to $<1 \%$ and the minimum score for modified peptides was set to $>40$.

Bioinformatics analysis, Gene Ontology (GO) annotation, domain annotation, Kyoto Encyclopedia of Genes and Genomes (KEGG) pathway annotation and subcellular localization. GO annotations $(28,29)$ of the proteome were derived from the UniProt-GOA database (www. http://www.ebi. ac.uk/GOA/, gaf-version, 2.0). If identified proteins were not annotated by the UniProt-GOA database, InterProScan software (http://www.ebi.ac.uk/interpro/,version:InterProScan 5.0) was used to annotate the GO functions of proteins based on a protein sequence alignment. For each category, a two-tailed Fisher's exact test was applied to detect enrichment of differentially expressed proteins against all identified proteins. GO terms with a corrected $\mathrm{P}<0.05$ were considered to be statistically significant. Descriptions of the functions of protein domains were annotated using InterProScan, a sequence analysis application, based on a protein sequence alignment prepared using the InterPro domain database (http://www.ebi. ac.uk/interpro/,version:InterProScan 5.0). The KEGG database was used to annotate protein pathways (30). First, the KEGG online service tool KEGG Automatic Annotation Server was employed to annotate protein descriptions in the KEGG database, after which the annotated results were mapped on the KEGG pathway database using the KEGG online service tool KEGG mapper. WoLF PSORT (https://www.genscript. com/wolf-psort.html), a subcellular localization predication software, was used to predict subcellular localization. WoLF PSORT is an updated version of PSORT/PSORT II for the prediction of eukaryotic sequences.
Motif analysis. The software Motif-x (http://motif-x.med. harvard.edu/) was used to model sequences containing modified amino acids at specific positions of 21-mers (10 amino acids upstream and downstream of the site) in all protein sequences. All protein sequences from the database were used as background database parameters and the default was used for other parameters.

Enrichment of GO terms, KEGG pathways and protein domain analysis. A two-tailed Fisher's exact test was used to determine enrichment of differentially expressed proteins compared with identified proteins. GO terms, KEGG pathways and protein domains with a corrected $\mathrm{P}<0.05$ were considered to be statistically significant.

\section{Results}

Analysis of crotonylated proteins. According to previous studies $(31,32)$, the present study used a fold-change of $<0.83$ to represent a downregulated protein and $>1.2$ to represent an upregulated protein, compared with the control group, to obtain accurate and appropriate data. The present study identified 353 crotonylated proteins from 770 crotonylated peptides, with 155 upregulated and 198 downregulated (Table SI). To identify an association between proteins and different expression multiples, these proteins were divided into four groups based on the fold changes in the levels of crotonylated sites: i) Q1 ( $0<$ ratio $<0.77 ; 381$ crotonylated peptides); ii) Q2 $(0.77<$ ratio $<0.83$; 80 crotonylated peptides); iii) Q3 $(1.2<$ ratio $<1.3$; 57 crotonylated peptides); and iv) Q4 (ratio $>1.3 ; 252$ crotonylated peptides). P-values were $<0.05$. The proteins from the quantiles were enriched with a cluster analysis. Proteins in Q2 and Q1 were downregulated, and proteins in Q4 and Q3 were upregulated. GO annotation can be divided into three primary categories: Biological processes, cellular components and molecular functions, which explain the biological function of proteins from different perspectives. Among the identified 353 crotonylated proteins from 770 crotonylated peptides, the distribution of the corresponding 353 proteins of the 770 crotonylated peptides in GO secondary annotations was determined. The most significantly enriched GO terms were as follows: i) Cellular components such as organelle, extracellular region and membrane, involving 972 proteins (with 69\%); ii) biological processes such as cellular process, single-organism process, biological regulation, response to stimulus and localization, involving 1,166 proteins (with 54\%); and iii) molecular functions such as binding and catalytic activity, involving 397 proteins $(75 \%)$. In total, $51 \%$ of these crotonylated proteins indicated a cytoplasmic localization involving 81 crotonylated proteins.

Functional enrichment of lysine crotonylation in GO terms, KEGG pathways and protein domains. Functional enrichment analysis of differential proteins was performed to assess the present GO term results. A reference data set was needed for functional enrichment, through which the statistically significant enrichment of a GO term can be identified. A two-tailed Fisher's exact test was used to determine enrichment of differential proteins in GO terms. The upregulated crotonylated proteins were mainly enriched in the cellular components of 
A \begin{tabular}{lllllllllll}
\multicolumn{10}{c}{$-\log 10$ (Fisher' exact test p value) } \\
0 & 0.5 & 1 & 1.5 & 2 & 2.5 & 3 & 3.5 & 4 & 4.5 & 5
\end{tabular}

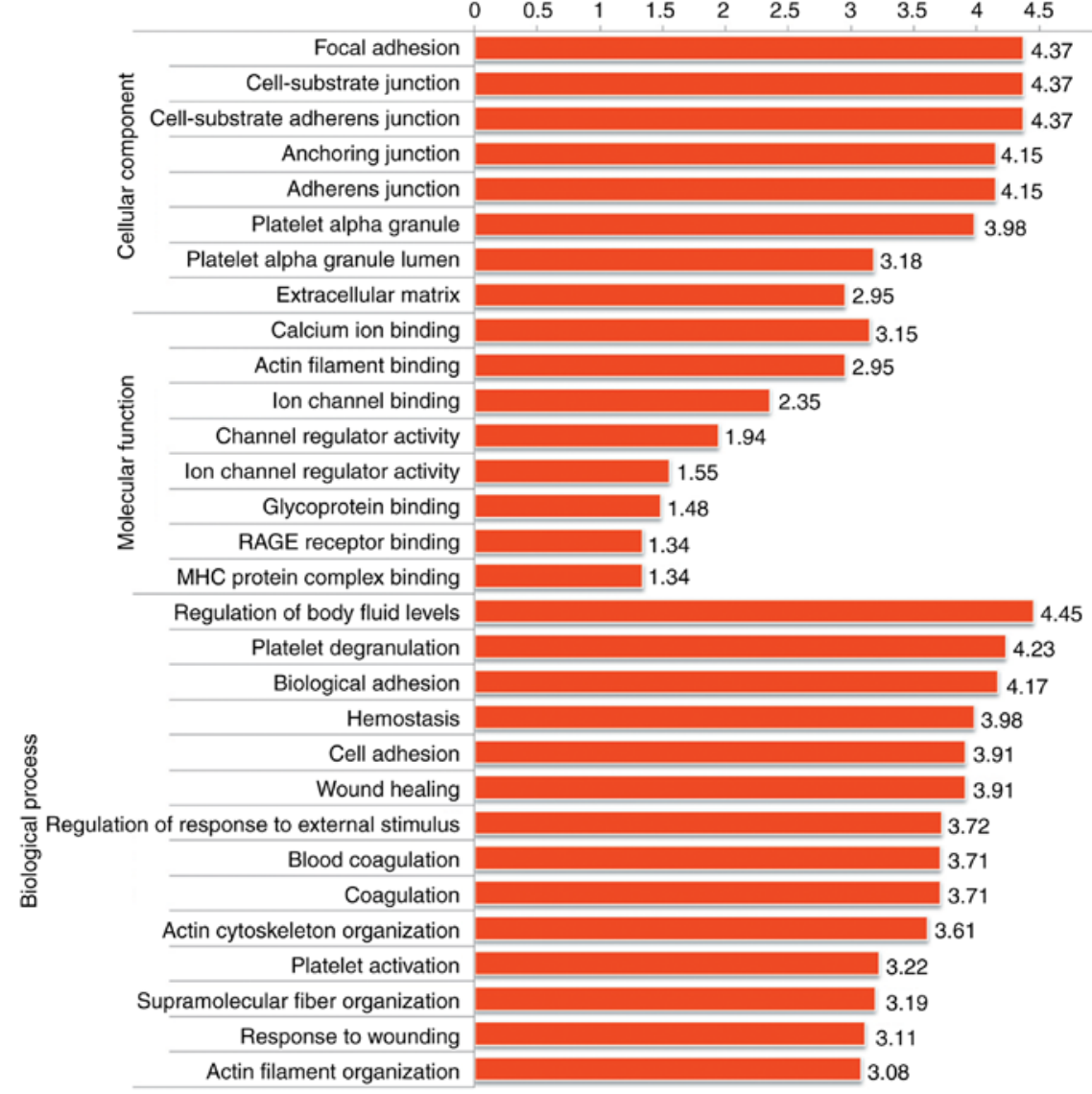

B

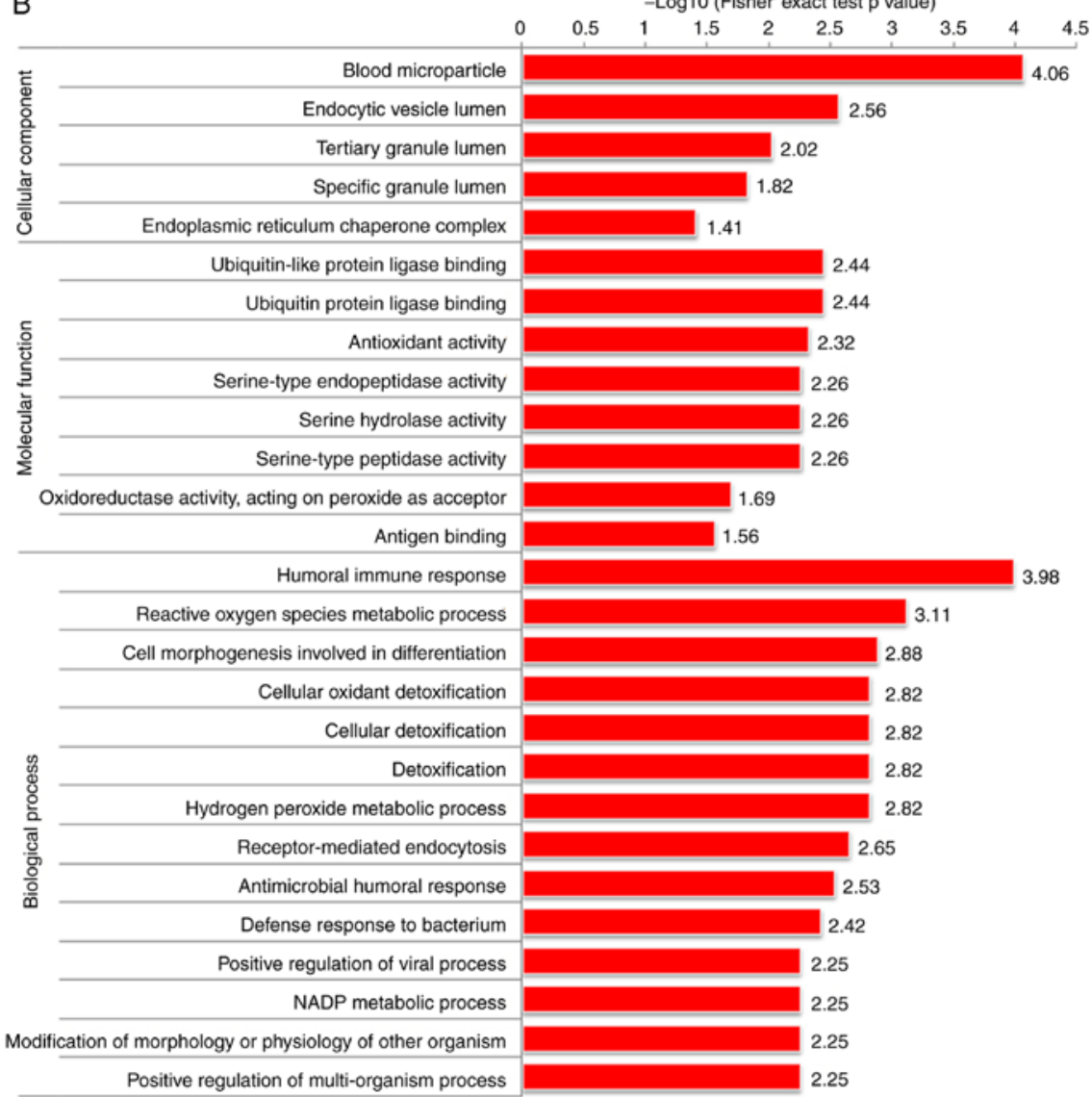

Figure 1. GO and KEGG analysis of upregulated and downregulated crotonylated proteins in immunoglobulin A nephropathy. (A) GO analysis of upregulated crotonylated proteins in the categories biological process, molecular function and cellular component. (B) GO analysis of downregulated crotonylated proteins in the categories biological process, molecular function and cellular component. 


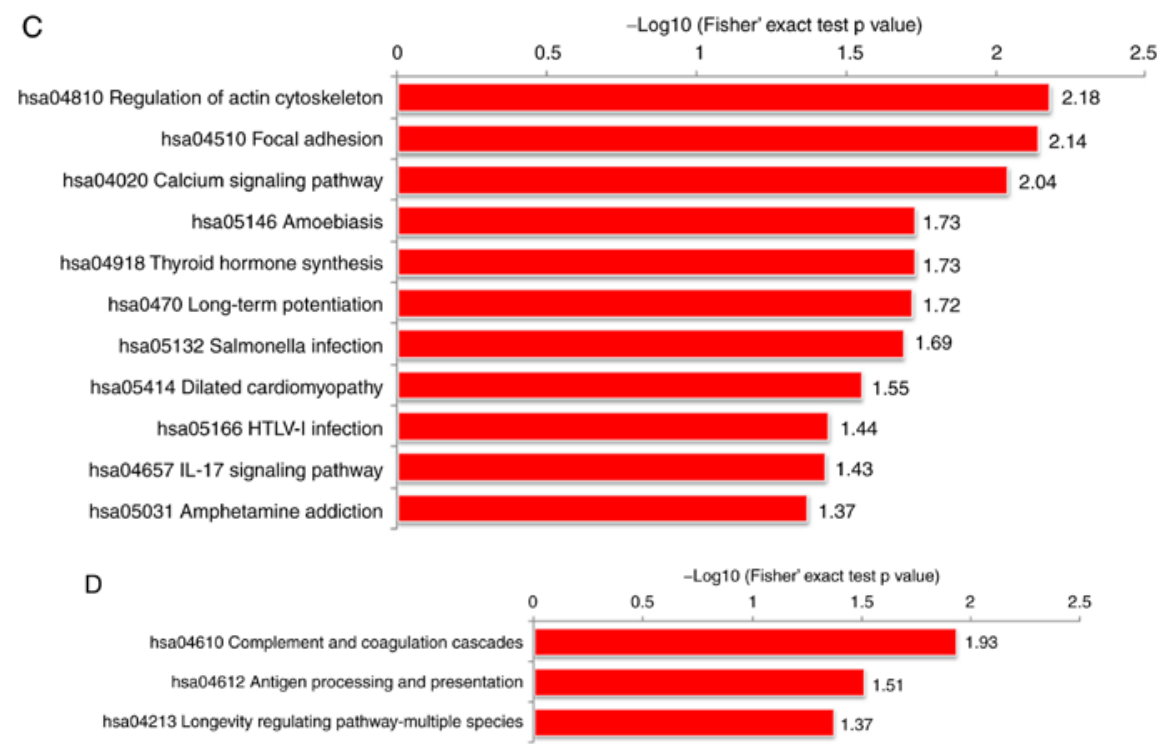

Figure 1. Continued. (C) KEGG analysis of upregulated crotonylated proteins. (D) KEGG analysis of downregulated crotonylated proteins. A corrected P $<0.05$ was considered to indicate a statistically significant result. GO, Gene Ontology; KEGG, Kyoto Encyclopedia of Genes and Genomes; MHC, major histocompatibility complex; IL-17, interleukin-17; HTLV-I, human T-cell lymphotropic virus type 1; RAGE, receptor for advanced glycation end products.

focal adhesion, cell-substrate junction, cell-substrate adherens junction, the biological process of regulation of body fluid levels and some molecular functions, including calcium ion binding (Fig. 1A). The downregulated crotonylated proteins were enriched in the cellular component of blood microparticle, the biological process of humoral immune response and some molecular functions, including ubiquitin-like protein ligase binding (Fig. 1B). According to the present KEGG-based functional enrichment analysis, the upregulated and downregulated crotonylated proteins were enriched in 11 and three important processes, respectively (Fig. 1C and D). Additionally, the present KEGG results of the antigen processing and presentation pathway (Fig. 2) suggested important roles for the crotonylated proteins in IgAN signaling mechanisms. Furthermore, upregulated crotonylated protein domains included the EF-hand domain, EF-hand domain pair, calponin homology domain and WD40-repeat-containing domain, suggesting a key role for lysine crotonylation in these domains. Conversely, specific protein domains displaying decreased crotonylation were not identified in the present study.

Cluster analysis of GO terms, KEGG pathways and protein domains. Cluster analyses of enriched GO terms, KEGG pathways and protein domains were performed to investigate additional information regarding the functions of the crotonylated proteins identified in the present study. Regarding GO terms, crotonylated proteins in Q1 were mainly involved in antigen binding and protein activation cascade, whereas the proteins in Q2 were suggested to be enriched in the regulation of protein complex assembly, positive regulation of protein complex assembly, cytoplasmic region and identical protein binding. Enrichment in negative regulation of protein metabolic process, modified amino acid binding, misfolded protein binding, cytoskeletal protein binding and protein complex binding of proteins in Q3 was identified, and proteins in Q4 may be involved in fibrinogen complex and actin filament (Fig. S1A-C).
Regarding KEGG pathways, proteins in Q1 were involved in complement and coagulation cascades, and those in Q2 in antigen processing and presentation. Proteins in Q3 were identified to be enriched in leukocyte transendothelial junctions, and the proteins in Q4 were suggested to be enriched in long-term potentiation and the interleukin-17 signaling pathway (Fig. S1D).

With regard to protein domains, Q1 crotonylated proteins showed enrichment in the thioredoxin domain, proteins in Q2 in the moesin tail domain, and proteins in Q4 in the myosin-like IQ motif and EF-hand domain (Fig. S1E). The present results suggested the possible mechanisms by which the activities of crotonylated proteins may be differentially regulated in IgAN .

Analysis of crotonylation sites in protein motifs. The present study analyzed sequence motifs to identify common sequences around crotonylation sites. The motifs of crotonylated peptides were detected using the Motif-x program and seven conserved motifs (KK, KD, AK, EK, K.D, KE, K.......K) were identified (Table I; Fig. 3A). The present results suggested motifs KK, KD and AK (Motif score $>15.00$ ) were conserved. Overall, the present results suggested that these motifs may reveal characteristics regarding crotonylation in patients with IgAN. Moreover, hierarchical cluster analysis was performed to analyze the motifs (Fig. 3B), and enrichment of charged A, D, $\mathrm{K}$ residues was observed at the -1 to +1 positions.

\section{Discussion}

IgAN predominantly affects young adults, but is also diagnosed in pediatrics and older individuals (33). Ig AN is diagnosed based on substantial accumulation of IgA immune complexes in mesangial cells, resulting in glomerular inflammation or kidney injury (19). The majority of patients with IgAN have notably high concentrations of circulating IgA1 (19). The current standardized method uses text and scoring of tissue injury in renal biopsies (19). However, a biopsy is invasive and can cause physical injury to patients with IgAN (19). 


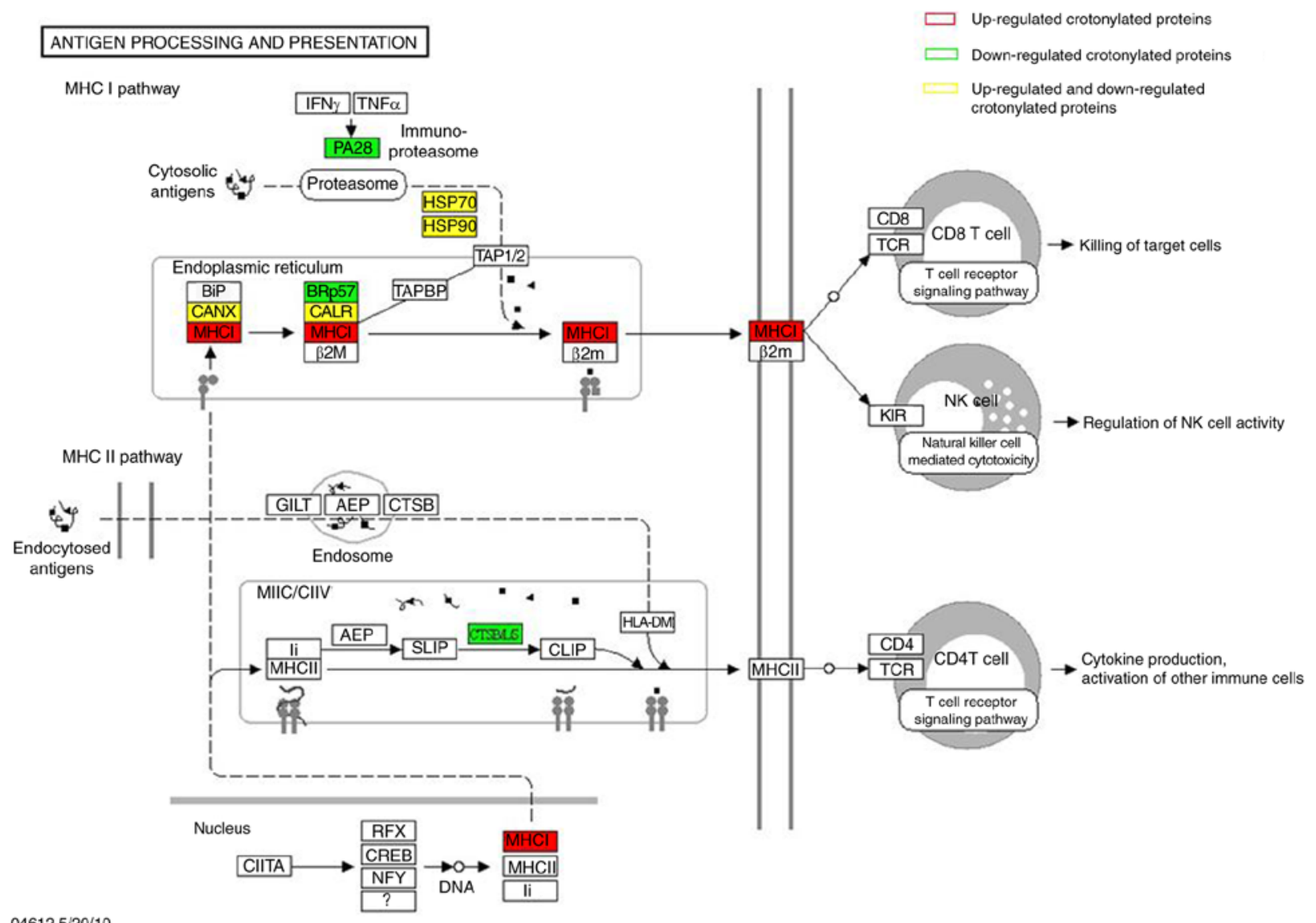

$046125 / 20 / 10$

(c) Kanehisa laboratories

Figure 2. Kyoto Encyclopedia of Genes and Genomes pathway antigen processing and presentation (KEGG copyright permission: 190342) results related to crotonylated proteins in immunoglobulin A nephropathy. Red represents upregulated crotonylated proteins, green represents downregulated crotonylated proteins, and yellow represents upregulated and downregulated crotonylated proteins.

A

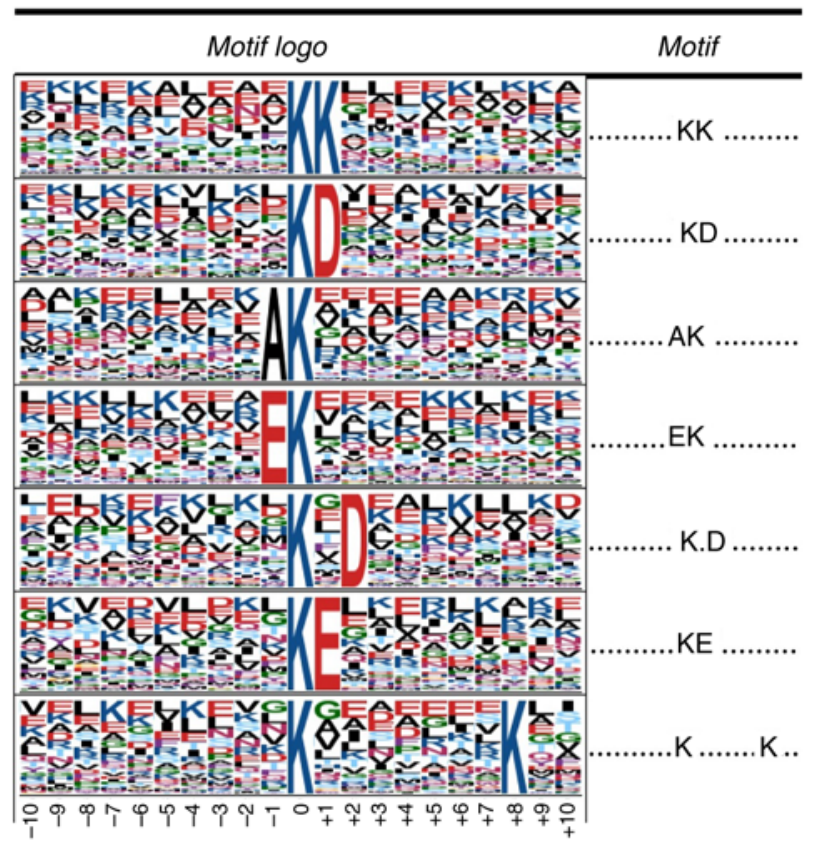

B

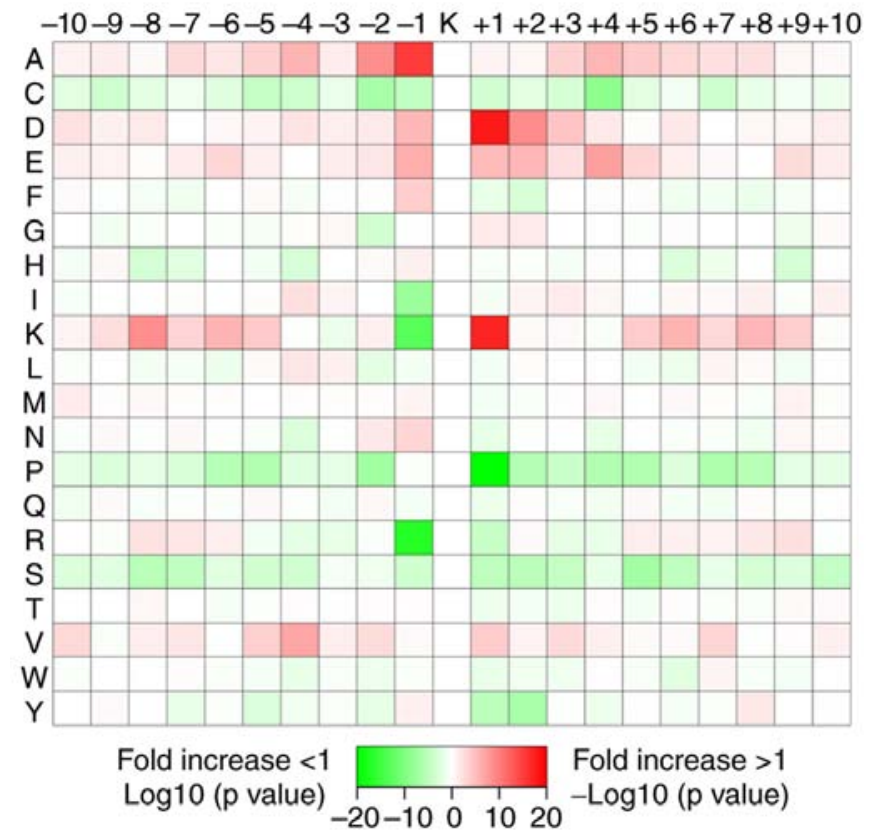

Figure 3. Motif analysis of all identified crotonylated sites in immunoglobulin A nephropathy. (A) Map showing the relative abundance of amino acids flanking crotonylated lysines. An intensity map showing the enrichment of amino acids at specific positions relative to crotonylated lysines is presented, ten amino acids downstream and upstream of the crotonylation site. (B) Heat map of the amino acid compositions around lysine crotonylation sites, suggesting the frequency of different types of amino acids around this residue. Red represents enrichment and green represents depletion. In total, seven enriched crotonylation site motifs were identified. 
Table I. Seven motifs retrieved from lysine crotonylated peptides.

\begin{tabular}{|c|c|c|c|c|c|c|}
\hline \multirow[b]{2}{*}{ Motif } & \multirow[b]{2}{*}{ Motif score } & \multicolumn{2}{|c|}{ Foreground } & \multicolumn{2}{|c|}{ Background } & \multirow[b]{2}{*}{ Fold increase } \\
\hline & & Matches & Size & Matches & Size & \\
\hline ..........KK....... & 16 & 179 & 1,169 & 47,867 & 602450 & 1.93 \\
\hline ...........KD ....... & 16 & 141 & 990 & 32,428 & 554583 & 2.44 \\
\hline .........AK....... & 15.48 & 113 & 849 & 30,225 & 522155 & 2.3 \\
\hline ..........EK ......... & 9.32 & 132 & 736 & 51,113 & 491930 & 1.73 \\
\hline ................ & 9.58 & 63 & 604 & 19,163 & 440817 & 2.4 \\
\hline ..........KE ........ & 7.82 & 97 & 541 & 42,246 & 421654 & 1.79 \\
\hline .................... & 6.97 & 63 & 444 & 26,675 & 379408 & 2.02 \\
\hline
\end{tabular}

PTMs alter protein function by regulating activity, domain structure and cellular localization (34). The present results identified 353 crotonylated proteins from 770 crotonylated peptides, and identified that these proteins mainly localize to the cytoplasm, nucleus, mitochondria and extracellular space. Thus, crotonylation may occur in a wide range of cellular proteins and may influence various biological processes in patients with $\operatorname{IgAN}$. In the present study, a large proportion of the crotonylated proteins $(n=381)$ clustered in Q1 and were regarded as downregulated. These crotonylated peptides may play important roles in $\operatorname{Ig} \mathrm{AN}$. The result of GO functional enrichment analysis indicated a role in the humoral immune response in patients with IgAN. In addition, KEGG pathway results of antigen processing and presentation suggest a predominant association for these crotonylated proteins in IgAN signaling mechanisms. A genome-wide association study (GWAS) in patients with IgAN was performed, identifying 15 common risk variants (34). Moreover, GWAS loci results revealed some pathogenic disease pathways that are probable novel therapeutic targets (35). The related pathways in patients with IgAN include antigen processing and presentation (36). Antigen processing and presentation by the $\mathrm{B}$ cell receptor promotes $\mathrm{T}$ cell-B cell interactions, which facilitate immunoglobulin class switching and affinity maturation (37). The pathways are regulated to influence the maturation and initiation of the humoral immune response (38). The generation of antigenic peptides is a complicated process mediated by numerous molecules that comprise the presentation and antigen processing machinery (39). Functional mitochondria have been shown to play a key role in antigen processing and presentation (40). KEGG pathway enrichment and clustering analyses were conducted in the present study to obtain additional information on the roles of lysine crotonylation. The results of the present study suggested that crotonylated proteins in Q2 were enriched in antigen processing and presentation, which may be related to the downregulation of crotonylated heat shock protein $90 \alpha$ family class A member 1 (HSP90AA1), HSPA8, HSPA1B, calreticulin (CALR), proteasome activator subunit 2 (PSME2) and HSPA5 proteins in patients with IgAN. HSPA1B stabilizes existing proteins and prevents aggregation, as well as mediating the folding of newly expressed proteins in the cytosol and organelles and it is a component of major histocompatibility complex III (https://www.genecards.org/cgi-bin/carddisp. pl?gene $=$ HSPA1B, provided by RefSeq, July 2008). HSPA8 is a chaperone protein with important roles in various cellular processes, including autophagy (41). Associations between CALR mutations and certain hematological parameters, including improved prognosis and a high platelet count in myeloproliferative neoplasms, have been observed (42). Single nucleotide polymorphisms in the HSP90AA1 gene influence the response of patients with systemic lupus erythematosus to glucocorticoid therapy (43). Additionally, the vascular endothelial growth factor (VEGF)/VEGFR2 pathway is related to hepatocellular carcinoma recurrence in patients who express HSPA8/HSP90AA1 at high levels (44). Moreover, an association between PSME2 and inflammatory responses has been observed in victims of suicide (45). In addition, HSPA5 is regarded as a potential biomarker of inflammatory myopathies (46). The present results suggested that upregulated crotonylated protein domains, such as the EF-hand domain, were in high abundance in patients with IgAN. The EF-hand domain was identified in a family of calcium-binding proteins, and is also present in every structural domain of the muscle protein troponin- $\mathrm{C}$ and the signaling protein calmodulin (47).

Although PTMs have been studied in various model systems, the biochemical function of lysine crotonylation in patients with $\operatorname{Ig} \mathrm{AN}$ remains poorly understood (48). The present results identified changes in crotonylation in patients with $\operatorname{IgAN}$. Although protein crotonylation may be related to IgAN, the present study cannot distinguish the progressive form from the benign form. The antigen processing and presentation processes related to the downregulation of crotonylated HSP90AA1, HSPA8, HSPA1B, CALR, PSME2 and HSPA5 proteins may suggest a predominant role for these proteins in IgAN signaling mechanisms. Furthermore, exposure of cultured tubular cells to inflammatory cytokine stimulation has revealed a key function of crotonylation in renal injury (49). The present results aid in understanding the role of crotonylation in the pathophysiological processes underlying IgAN. However, additional studies are needed to further investigate the functions of crotonylated proteins in patients with IgAN. The development of non-invasive diagnostic methods are important for assessing and monitoring disease progression, disease activity and therapeutic effects. 


\section{Acknowledgements}

Not applicable.

\section{Funding}

The present study was supported by The National Natural Science Foundation of China (grant no. 81671596), The Science and Technology Plan of Shenzhen (grant no. JCYJ201 60422164313440), The Natural Science Foundation of Guangxi (grant no. 2017GXNSFAA198375), The National Science Foundation for Young Scientists of China (grant no. 31700795) and The Research and Technology Development Plan of Guilin Scientific and Technological Innovation Ability and Condition Construction (grant no. 20170117-1).

\section{Availability of data and materials}

The datasets used and/or analyzed during the current study are available from the corresponding author on reasonable request.

\section{Authors' contributions}

WD, WS and YD designed the search strategy. HL, JC, YZo and DT were involved in data analysis. YX, YZh, GZ and MO were involved in acquisition of data and drafting the manuscript. WX, RZ and FZ were involved in interpretation of data and revising the manuscript critically for important intellectual content. All authors have reviewed the final manuscript before submission and given final approval of the version to be published.

\section{Ethics approval and consent to participate}

Informed consent was obtained from all participants. The study was performed in compliance with the Declaration of Helsinki and was approved by The 924th Hospital Ethics Committee.

\section{Patient consent for publication}

Not applicable.

\section{Competing interests}

The authors declare that they have no competing interests.

\section{References}

1. Jenuwein $\mathrm{T}$ and Allis CD: Translating the histone code. Science 293: 1074-1080, 2001.

2. Zamdborg L, Leduc RD, Glowacz KJ, Kim YB, Viswanathan V, Spaulding IT, Early BP, Bluhm EJ, Babai S and Kelleher NL: ProSight PTM 2.0: Improved protein identification and characterization for top down mass spectrometry. Nucleic Acids Res 35: W701-W706, 2007.

3. Witze ES, Old WM, Resing KA and Ahn NG: Mapping protein post-translational modifications with mass spectrometry. Nat Methods 4: 798-806, 2007.

4. Olsen JV and Mann M: Status of large-scale analysis of post-translational modifications by mass spectrometry. Mol Cell Proteomics 12: 3444-3452, 2013.

5. Tan M, Luo H, Lee S, Jin F, Yang JS, Montellier E, Buchou T, Cheng Z, Rousseaux S, Rajagopal N, et al: Identification of 67 histone marks and histone lysine crotonylation as a new type of histone modification. Cell 146: 1016-1028, 2011.
6. Montellier E, Rousseaux S, Zhao Y and Khochbin S: Histone crotonylation specifically marks the haploid male germ cell gene expression program post-meiotic male-specific gene expression. Bioessays 34: 187-193, 2012.

7. Li Y, Sabari BR, Panchenko T, Wen H, Zhao D, Guan H, Wan L, Huang H, Tang Z, Zhao Y, et al: Molecular coupling of histone crotonylation and active transcription by AF9 YEATS domain. Mol Cell 62: 181-193, 2016.

8. Sabari BR, Tang Z, Huang H, Yong-Gonzalez V, Molina H, Kong HE, Dai L, Shimada M, Cross JR,Zhao Y, et al: Intracellular crotonyl-CoA stimulates transcription through p300-catalyzed histone crotonylation. Mol Cell 58: 203-215, 2015.

9. Liu S, Yu H, Liu Y, Liu X, Zhang Y, Bu C, Yuan S, Chen Z, Xie G, Li W, et al: Chromodomain protein CDYL Acts as a Crotonyl-CoA hydratase to regulate histone crotonylation and spermatogenesis. Mol Cell 67: 853-866.e5, 2017.

10. Gajjala PR, Fliser D, Speer T, Jankowski V and Jankowski J: Emerging role of post-translational modifications in chronic kidney disease and cardiovascular disease. Nephrol Dial Transplant 30: 1814-1824, 2015.

11. Ruiz-Andres O, Sanchez-Niño MD, Cannata-Ortiz P, RuizOrtega M, Egido J, Ortiz A and Sanz AB: Histone lysine crotonylation during acute kidney injury in mice. Dis Model Mech 9: 633-645, 2016.

12. Fontecha-Barriuso M, Martin-Sanchez D, Ruiz-Andres O, Poveda J, Sanchez-Niño MD, Valiño-Rivas L, Ruiz-Ortega M, Ortiz A and Sanz AB: Targeting epigenetic DNA and histone modifications to treat kidney disease. Nephrol Dial Transplant 33: 1875-1886, 2018.

13. Zaza G, Bernich P and Lupo A; 'Triveneto' Register of Renal Biopsies (TVRRB): Incidence of primary glomerulonephritis in a large north-eastern Italian area: A 13-year renal biopsy study. Nephrol Dial Transplant 28: 367-372, 2013.

14. Otani M, Nakata J, Kihara M, Leroy V, Moll S, Wada Y and Izui S: O-glycosylated IgA rheumatoid factor induces IgA deposits and glomerulonephritis. J Am Soc Nephrol 23: 438-446, 2012.

15. Li LS and Liu ZH: Epidemiologic data of renal disease from a single unit in China: Analysis based on 13,519 renal biopsies. Kidney Int 66: 920-923, 2004.

16. Suzuki H, Kiryluk K, Novak J, Moldoveanu Z, Herr AB, Renfrow MB, Wyatt RJ, Scolari F, Mestecky J, Gharavi AG and Julian BA: The pathophysiology of IgA nephropathy. J Am Soc Nephrol 22: 1795-1803, 2011.

17. Maixnerova D, Reily C, Bian Q, Neprasova M, Novak J and Tesar V: Markers for the progression of IgA nephropathy. J Nephrol 29: 535-541, 2016.

18. Geddes CC, Rauta V, Gronhagen-Riska C, Bartosik LP, Jardine AG, Ibels LS, Pei Y and Cattran DC: A tricontinental view of IgA nephropathy. Nephrol Dial Transplant 18: 1541-1548, 2003.

19. Zhang C, Zeng X, Li Z, Wang Z and Li S: Immunoglobulin A nephropathy: Current progress and future directions. Transl Res 166: 134-144, 2015.

20. Tan K, Chen J, Li W, Chen Y, Sui W, Zhang Y and Dai Y: Genome-wide analysis of microRNAs expression profiling in patients with primary IgA nephropathy. Genome 56: 161-169, 2013.

21. Serino G, Sallustio F, Cox SN, Pesce F and Schena FP: Abnormal miR-148b expression promotes aberrant glycosylation of IgA1 in IgA nephropathy. J Am Soc Nephrol 23: 814-824, 2012.

22. Vuong MT, Hahn-Zoric M, Lundberg S, Gunnarsson I, van Kooten C, Wramner L, Seddighzadeh M, Fernström A, Hanson LÅ, Do LT, et al: Association of soluble CD89 levelswith disease progression but not susceptibility in $\operatorname{IgA}$ nephropathy. Kidney Int 78: 1281-1287, 2010.

23. Peters HP, van den Brand JA and Wetzels JF: Urinary excretion of low-molecular-weight proteins as prognostic markers in IgA nephropathy. Neth J Med 67: 54-61, 2009.

24. Ebefors K, Liu P, Lassén E, Elvin J, Candemark E, Levan K, Haraldsson B and Nyström J: Mesangial cells from patients with IgA nephropathy have increased susceptibility to galactose-deficient IgA1. BMC Nephrol 17: 40, 2016.

25. Liang M: Establishment of reference intervals for serum creatinine concentrations of the children and the adults in shaoxing area by sarcosine oxidase assay. J Med Reaserch 41: 117-119, 2012.

26. Wang $\mathrm{F}$ and Zhang YH: Relationship of cystatin C, fibrinogen, and 24-hour urinary protein with renal pathological grade in children with Henoch-Schönlein purpura nephritis. Zhongguo Dang Dai Er Ke Za Zhi 18: 233-237, 2016. 
27. Tyanova S, Temu T and Cox J: The MaxQuant computational platform for mass spectrometry-based shotgun proteomics. Nat Protoc 11: 2301-2319, 2016.

28. Ashburner M, Ball CA, Blake JA, Botstein D, Butler H, Cherry JM, Davis AP, Dolinski K, Dwight SS, Eppig JT, et al: Gene ontology: Tool for the unification of biology. The gene ontology consortium. Nat Genet 25: 25-29, 2000.

29. The Gene Ontology Consortium: The Gene Ontology Resource: 20 years and still GOing strong. Nucleic Acids Res 47: D330-D338, 2019.

30. Kanehisa M and Goto S: KEGG: Kyoto encyclopedia of genes and genomes. Nucleic Acids Res 28: 27-30, 2000.

31. Luu LDW, Octavia S, Zhong L, Raftery MJ, Sintchenko V and Lan R: Proteomic adaptation of australian epidemic bordetella pertussis. Proteomics 18: e1700237, 2018

32. Du M,Liu X, Ma N, Liu X, Wei J, Yin X, Zhou S, Rafaeli A, Song Q and An S: Calcineurin-mediated dephosphorylation of Acetyl-coA carboxylase is required for pheromone biosynthesis activating neuropeptide (PBAN)-induced sex pheromone biosynthesis in helicoverpa armigera. Mol Cell Proteomics 16: 2138-2152, 2017.

33. Magistroni R, D'Agati VD, Appel GB and Kiryluk K: New developments in the genetics, pathogenesis, and therapy of $\operatorname{Ig} \mathrm{A}$ nephropathy. Kidney Int 88: 974-989, 2015.

34. Wu Z, Cheng Z, Sun M, Wan X, Liu P, He T, Tan M and Zhao Y: A chemical proteomics approach for global analysis of lysine monomethylome profiling. Mol Cell Proteomics 14: 329-339, 2015

35. Gharavi AG, Kiryluk K, Choi M, Li Y, Hou P, Xie J, Sanna-Cherchi S, Men CJ, Julian BA, Wyatt RJ, et al: Genomewide association study identifies susceptibility loci for $\operatorname{IgA}$ nephropathy. Nat Genet 43: 321-327, 2011

36. Kiryluk K, Li Y, Scolari F, Sanna-Cherchi S, Choi M, Verbitsky M, Fasel D, Lata S, Prakash S, Shapiro S, et al: Discovery of new risk loci for IgA nephropathy implicates genes involved in immunity against intestinal pathogens. Nat Genet 46: 1187-1196, 2014.

37. Jensen PE: Recent advances in antigen processing and presentation. Nat Immunol 8: 1041-1048, 2007.

38. Katkere B, Rosa S and Drake JR: The Syk-binding ubiquitin ligase $\mathrm{c}-\mathrm{Cbl}$ mediates signaling-dependent $\mathrm{B}$ cell receptor ubiquitination and B cell receptor-mediated antigen processing and presentation. J Biol Chem 287: 16636-16644, 2012.

39. Goldberg AL, Cascio P, Saric T and Rock KL: The importance of the proteasome and subsequent proteolytic steps in the generation of antigenic peptides. Mol Immunol 39: 147-164, 2002
40. Bonifaz LC, Arzate S and Moreno J: Endogenous and exogenous forms of the same antigen are processed from different pools to bind MHC class II molecules in endocytic compartments. Eur J Immunol 29: 119-131, 1999.

41. Stricher F, Macri C, Ruff M and Muller S: HSPA8/HSC70 chaperone protein: Structure, function, and chemical targeting. Autophagy 9: 1937-1954, 2013.

42. Luo W and Yu Z: Calreticulin (CALR) mutation in myeloproliferative neoplasms (MPNs). Stem Cell Investig 2: 16, 2015.

43. Zou YF, Xu JH, Gu YY, Pan FM, Tao JH, Wang DG, Xu SQ, Xiao H, Chen PL, Liu S, et al: Single nucleotide polymorphisms of HSP90AA1 gene influence response of SLE patients to glucocorticoids treatment. Springerplus 5: 222, 2016.

44. Xiang X, You XM and Li LQ: Expression of HSP90AA1/HSPA8 in hepatocellular carcinoma patients with depression. Onco Targets Ther 11: 3013-3023, 2018.

45. Galfalvy H, Haghighi F, Hodgkinson C, Goldman D, Oquendo MA, Burke A, Huang YY, Giegling I, Rujescu D, Bureau A, et al: A genome-wide association study of suicidal behavior. Am J Med Genet B Neuropsychiatr Genet 168: 557-563, 2015.

46. Xiao F, Tan JZ, Xu XY and Wang XF: Increased levels of HSPA 5 in the serum of patients with inflammatory myopathies-preliminary findings. Clin Rheumatol 34: 715-720, 2015.

47. Perret $\mathrm{C}$, Lomri $\mathrm{N}$ and Thomasset M: Evolution of the 'EF-hand' family of calcium-binding proteins. Adv Exp Med Biol 269: 17-20, 1990

48. Qiu WR, Sun BQ, Xiao X, Xu ZC, Jia JH and Chou KC: iKcr-PseEns: Identify lysine crotonylation sites in histone proteins with pseudo components and ensemble classifier. Genomics 110: 239-246, 2018.

49. Ruiz-Andres O, Suarez-Alvarez B, Sánchez-Ramos C Monsalve M, Sanchez-Niño MD, Ruiz-Ortega M, Egido J, Ortiz A and Sanz AB: The inflammatory cytokine TWEAK decreases PGC-1 $\alpha$ expression and mitochondrial function in acute kidney injury. Kidney Int 89: 399-410, 2016.

This work is licensed under a Creative Commons Attribution-NonCommercial-NoDerivatives 4.0 International (CC BY-NC-ND 4.0) License. 